\title{
Design and Implementation of 6LoWPAN Application: A Performance Assessment Analysis
}

\author{
Nin Hayati Mohd Yusoff ${ }^{1}$, Nurul Azma Zakaria ${ }^{2}$ \\ Centre for Advanced Computing Technology (C-ACT) \\ Fakulti Teknologi Maklumat Dan Komunikasi \\ Universiti Teknikal Malaysia, Melaka, Malaysia
}

\author{
Adil Hidayat Rosli ${ }^{3}$ \\ My6 Initiative Berhad 897128-T \\ 1-21-01Sunteh @Penang Cybercity \\ Penang, Malaysia
}

\begin{abstract}
Industrial Revolution 4.0 promises an overall improvement to the communications technology by improving on the quality and flexibility of IoT application deployment. Currently, most of these applications are embedded devices from various manufacturers, networks, and technologies. As such, it would be total chaos of just getting the various and myriad devices and technologies to work together, let alone making them to work in perfect harmony. Regardless, the IoT espouses on the seamless integration and interoperability of the said devices and technologies. In realizing this goal, it would be imperative to say that the ability of the IoT system in adopting and adapting to new devices, services, and application is crucial, while at the same time it would not be in any way jeopardizing or compromising the existing system, especially the routing protocol. In view of the IP-based communication technology in WSN, the 6LoWPAN network has been chosen for the task, and the RPL protocol has been strongly considered for the 6LoWPAN solution. However, the RPL overhead tends to be spiralling upwards when additional information transmission occurs. In mitigating this anomaly, therefore, the HRPL was proposed to enhance the RPL protocol in reducing routing overhead. This study focusses on the performance analysis of RPL and HRPL based on the physical experimentation of the 6LoWPAN network in a real scenario. The results show HRPL protocol outperforms in all the performance-tested evaluations: CTO (38.7\%), latency (26\%), and convergence time (37\%). It was also discovered that the number of DIS and DAO (RPL control message) packet is significantly reduced when the DIO message was reduced. At the same time, latency and convergence time also registered a decrease in their respective values correspondingly. Meanwhile, based on our observation, several experiments are needed to investigate how variants topology affect HRPL capabilities.
\end{abstract}

Keywords-6LoWPAN protocol; performance analysis; overhead

\section{INTRODUCTION}

IPv6 Low Power Area Network (6LoWPAN) is one of the IP-based communication technologies in WSN [1]. It allows a huge number of embedded devices to be connected directly to the internet (end to end communication) by using the large available IPv6 address space [2][3][4]. In these technologies, the transmission packets from a source node to destinations node over IEEE 802.15.4 based network using the multi-hop communication as we known wireless mesh network. Thus, the energy used for connectivity will save especially for data and information exchange through features of various metrics, including low bandwidth, different topologies (star, mesh, etc.), low power consumption, low-end node device cost, and scalable networks. Those, 6LoWPAN an essential part of the IoT development has been an explorer in [3] [5][6], especially for home automation systems. However, the current limitations of 6LoWPAN, such as a small frame length size could complicate to implement in real-life as has been discussed in [7]. The 6LoWPAN frame length for each data transmission limited to only 127 bytes as compared to IPv6's Maximum Transmission Unit (MTU), which is 1280 bytes. Therefore, for the successful transmission of packet frames, various techniques like header compressor, fragmentation, and reassembly technique are used to compress the User Datagram Protocol (UDP) header and IPv6 header. Unfortunately, in [8] and [9] state those techniques would affect the high packet reading of control traffic overhead. This issue contributes to the challenges in designing 6LoWPAN routing protocols that could provide efficient Quality of Services QoS [10] which ensures the network services are reliable for different users and applications [11] [12].

The IETF has proposed two working groups, Routing Over Low Power and Lossy (ROLL) networks and mobile ad hoc networks (MANET) to develop the 6LoWPAN routing algorithm requirement. Fig. 1 presents the multipath routing protocols and the taxonomy in 6LoWPAN.

According to [14] [8][15], RPL (from the ROLL working group) is effective, and simplicity has made it a strong candidate for the 6LoWPAN routing protocol. However, to suit into 6LoWPAN environments, some modifications with the effective technique are required especially in control traffic overhead. Based on a study in [12], the authors perform a detailed comparison of RPL, Ad-hoc On-demand Distance Vector (AODV), and Dynamic MANET On-demand (DYMO), exposing the network topology change, routing overhead and latency. It was found the performance of RPL is better than AODY and DYMO in terms of latency and throughputs, but obviously, RPL overhead is higher than AODY and DYMO. Besides, in 2016, [16] [17] in their study state that the RPL is effective in term of very fast network set up when implementing in 6LoWPAN but need further improved solution in overhead. Furthermore, the majority of the existing study of RPL protocol does not test in 6LoWPAN protocol stack and very limited study test real scenarios such as smart home automation. Therefore, it is necessary to study the RPL overhead to enhance the RPL protocol in order to achieve an efficient performance of QoS in real scenario 6LoWPAN network. 


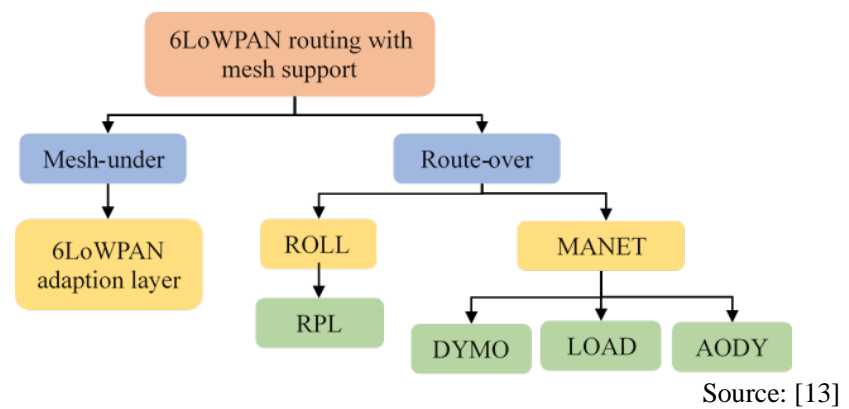

Fig. 1. Multipath Routing Protocol Taxonomy.

Due to this matter, we conduct the intensive study via document analysis to investigate the factors that can define the causes of RPL routing overhead in 6LoWPAN [18]. We show that RPL routing overhead increased in three scenarios: i) Network topology change, ii) Additional information transmission, iii) Speed movement of node increased. As a result, a new RPL implementation for 6LoWPAN has been developed, namely HRPL, to enhance RPL that can minimize the routing overhead and provides an efficient way of communicating devices. As an extension of the work presented in [19], this paper we address the to analyze the performance of HRPL in a real application, an experiment platform based on 6LoWPAN environment has been designed and implemented. Hence, to assess the effectiveness of HRPL in the 6LoWPAN environment (grid topology) in three QoS performance metrics, there are 1) latency, 2) control traffic overhead, and 3) convergence time. Besides, in paper [20] mentioned the grid topology is considered as an advantage regarding scalability in exchange for the data between the node.

The reminders of this paper are organized as follows: Section II provided the explanations of routing protocol (RPL and HRPL), the methodology in testbed development, and the performance metric used. Result and discussion, we present in Section III, and finally, conclusion and futures work in Section IV.

\section{MATERIAL AND METHOD}

\section{A. Routing Protocol}

1) $R P L$ : RPL protocol is a distance-vector that introduced for Low-Power and Lossy Networks (LLNs). RPL builds network topology called Destination-Oriented Directed Acyclic Graph (DODAG) using the objective function (OF) in selecting the best path and Directed Acyclic Graph (DAG). In fact, RPL has calculated the link in a network based on the least cost route between any two nodes is the route with distance and the cost of reaching a destination using Minimum Rank with Hysteresis Objective Function (MRHOF) that use minimum Expected Transmission Count (ETX) metrics or Objective Function Zero (OF0) that use minimum multi-hop metrics. To control the construction and maintenance of network topology, RPL generated three types of control message: i) Destination-Oriented Directed Acyclic Graphs (DODAG) Information Object (DIO), ii) DODAG Information Solicitation (DIS), and iii) Destination Advertisement Object (DAO). Fig. 2 illustrates the DODAG structure, Fig. 3 shows the RPL control message sequence scenario with layer registration, Fig. 4 presents the process message flow that implements in this paper. DIS message is used for soliciting the DIO message to make a quick response to join the DODAG. First, Root Nodes (RN) broadcast the DIOs to select the root as their parent (upward route) and computes its RANK. The nodes receiving the DIOs message that contain information about RPL instance, compute the DODAGID rank, and DODAGVersionNumber allows to constructs and maintains the DODAG route. Then the RN setting as Parent Node(PN)/Intermediate Node (IN) (6LoWPAN Router (6LR) those 6LoWPAN Nodes (6LN) would re-broadcast the DIOs to the further nodes (Child Node $(\mathrm{CN})$ ) or Leaf Node (LN)). DAO messages are maintaining the downward routes while DAO Acknowledgement (DAO$\mathrm{ACK}$ ) is a unicast message in response to the DAO. These proceed repeat such as the way DODAG topology is constructed.

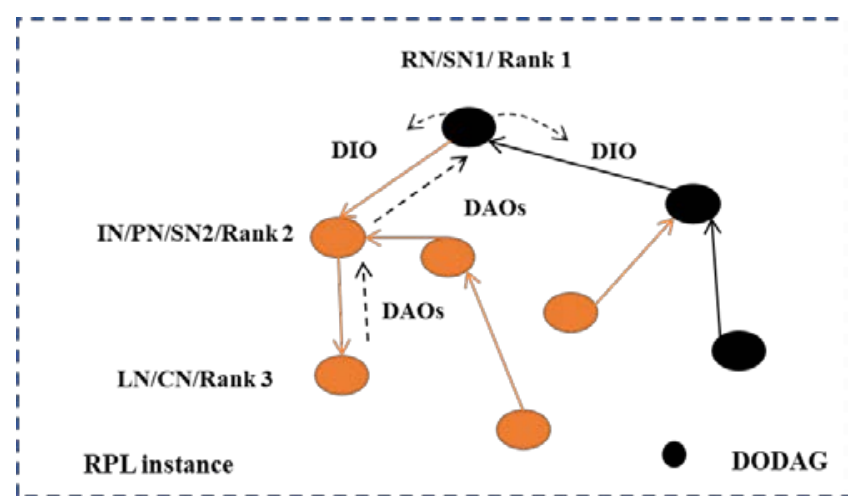

Fig. 2. RPL Topology (DODAG Structure).

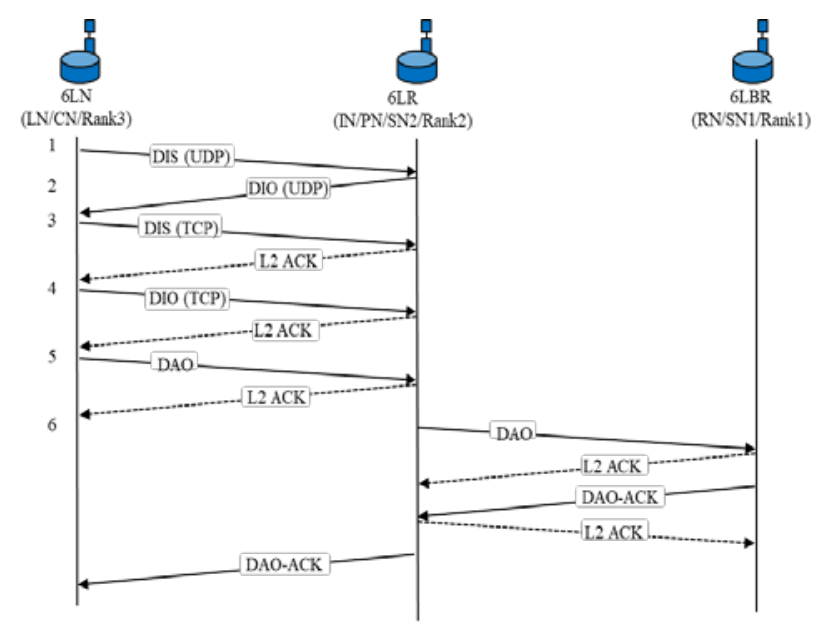

Fig. 3. RPL Routing Layer Message Process. 


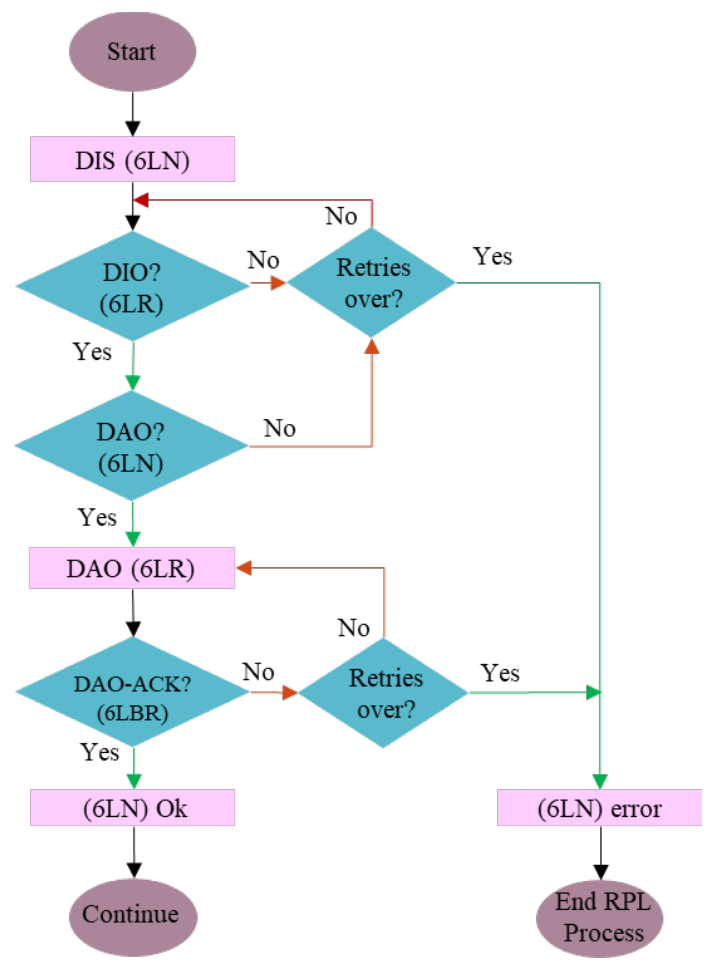

Fig. 4. RPL Flowchart.

2) HRPL: HRPL was proposed to enhance the RPL. HRPL was introduced to reduce the Control Traffic Overhead (CTO) caused by redundant transmission. We introduced the T-Cut Off Delay to set the limit of delay and $\mathrm{H}$ field to respond on actions taken within the T-Cut Off Delay. As presented in Fig. 5, RN broadcast the DIO message to join the DODAG at the same time, the algorithm calculates and sets the $\mathrm{T}$ Cut Off Delay for the RN. If the RN receives duplicate DIO messages from other nodes before T-Cut Off Delay expires, the $\mathrm{H}$ field is a response based on three conditions: i) rebroadcast the DIO message) or ii) discard the DIO message or iii) to make no changes. However, when the T-Cut Off Delay expires the nodes must rebroadcast the DIOs messages. The addition of the $\mathrm{H}$ field is expected to reduce the control traffic overhead at the same time maximize the network lifetime, and minimize the latency.

\section{B. Method}

This research focuses on evaluating the performance of HRPL and RPL protocol in real sensor nodes (SN) based on the 6LoWPAN environment. We compare the existing RPL protocol with our proposed protocol HRPL in terms of control traffic overhead, latency, and convergence time. Fig. 6 illustrates the testing and validation design in this analysis.

\section{Building the Testbed Scenario}

For the validity of our approach, we use a Texas Instruments (TI) CC2538 wireless controller system-on-chip for 6LoWPAN application compliant 2.5-GHz IEEE 802.15.4. Table I shows the list of equipment required for a physical deployment scenario. All the work on the development of the 6LoWPAN testbed is presented in Fig. 7. The development process involves the deployed hardware infrastructure, physical network, and integrated with software services for managing and controlling the hardware.

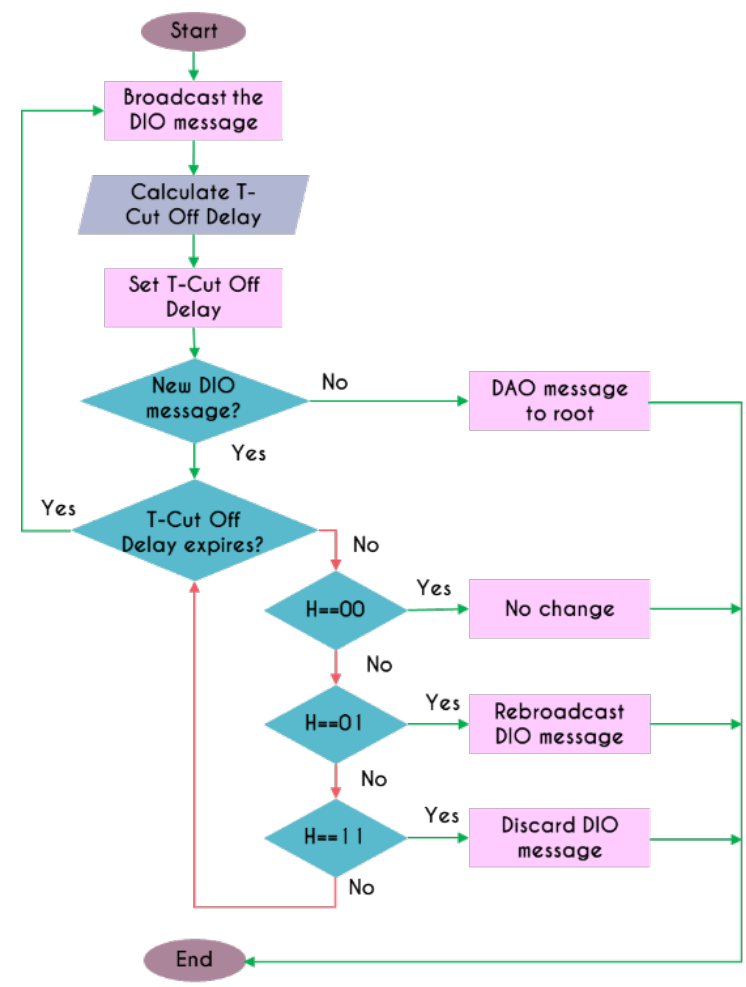

Fig. 5. HRPL Flowchart.

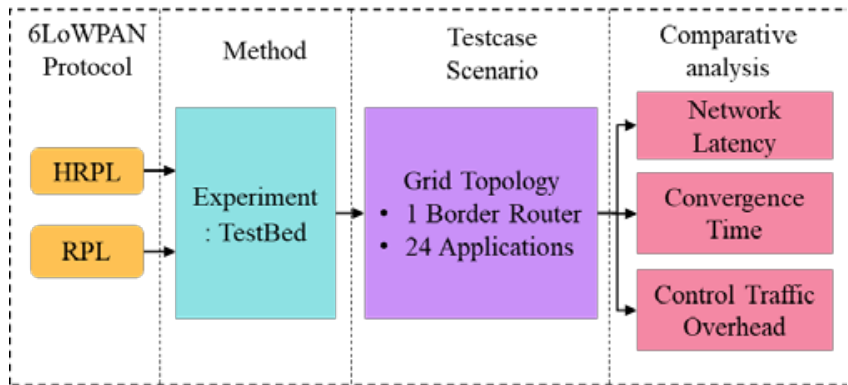

Fig. 6. Testing and Validity Design.

TABLE I. 6LOWPAN TESTBED EQUIPMENT

\begin{tabular}{|c|c|c|}
\hline \multicolumn{3}{|c|}{ Hardware Equipment } \\
\hline Equipment & Count & Types and Description \\
\hline Sensor Node & 24 & - $\quad$ The ARM $®$ Cortex ${ }^{\mathrm{TM}}-\mathrm{M} 3$ processor, \\
\hline Border Router & 1 & IEEE 802.15.4. \\
\hline Sniffer & 1 & $\begin{array}{l}\text { - CC2531 USB Dongle - Fully } \\
\text { operational USB device that can be } \\
\text { plugged into a PC } \\
2 \text { x LEDs } \\
2 \text { x pushbuttons } \\
\text { USB A connector for PC } \\
\text { 10-pin program/debug header } \\
\text { PCB antenna (meandered inverted F) } \\
\text { Connector pads for external sensors }\end{array}$ \\
\hline
\end{tabular}




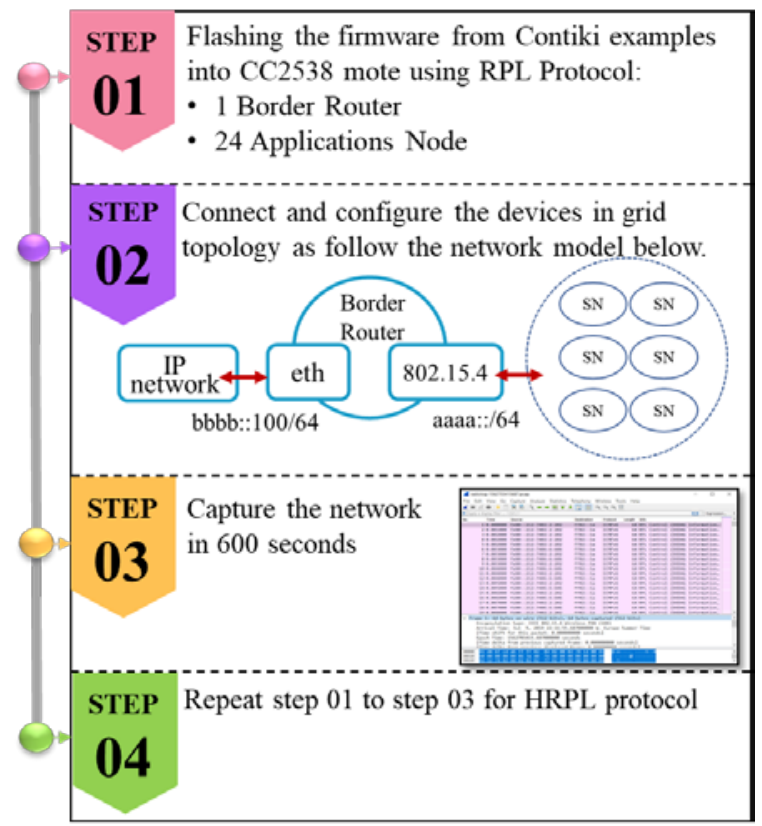

Fig. 7. The Procedure for Development of 6LoWPAN Testbed.

First, a modified firmware from the Contiki example is flashed into the CC2538 mote. One of the motes is used as a Border Router (BR) (router mode) while the rest of the motes are flashed as an SN. The firmware parameter setup for HRPL and RPL are presented in Table II. For this experiment, the default trickle algorithm [21] used to control the amount of DIO message in routing traffic by setting the parameter of DIO Interval Min as $12 \mathrm{~ms}$ and DIO interval doublings as $8 \mathrm{~ms}$. To achieve the best Packet Delivery Ratio (PDR), the Minimum Rank with Hysteresis Objective Function (MRHOF, RFC6719) with Expected Transmission Count (ETX, RFC 6551) metrics are used as path selection mechanism [22][23].

In the next step, the BR running the 6LBR software will act as a proxy/translator between the 802.15.4 network and the LAN network. The 6LBR can be operated as a smart bridge, router, or a transparent bridge, which in this experiment is being configured as a router. The 802.15.4 network is assigned the IPv6 subnet of aaaa::/64 while the LAN network is assigned the bbbb::/64. Fig. 8 shows the 6LoWPAN testbed using the CC2538 scenario where the nodes are deployed with grid topology in an indoor environment. Fig. 9 displays the 6LBR information and Fig. 10 exhibits the SN information in the webserver. The configuration setting of the 6LBR parameter as presented in Table III.

The RPL/HRPL control messages (DIS, DIO, DAO, DAOACK) are received when NDP configures the RPL/HRPL network. We observed the RPL control message to analyze 6LoWPAN packets by running a 6LoWPAN traffic sniffer and the Wireshark packet capture tool, as shown in Fig. 11. We recorded the capture after 30 minutes of the experiment running [24] and the observation time is 30 minutes. For energy monitoring, only the energy consumption of the radio transceivers is monitored based on the chip's manufacturer datasheets as shown in Table IV. We ignored other small energy consumptions such as sensors, actuators, and microcontrollers.
TABLE II. SYSTEM PARAMETER FOR CC2538 MOTE FIRMWARE

\begin{tabular}{|l|l|}
\hline 6LoWPAN Protocol & HRPL compared with RPL \\
\hline Physical (PHY) and Data Link & $\begin{array}{l}\text { IEEE 802.15.4 PHY } \\
\text { IEEE 802.15.4 MAC and CSMA }\end{array}$ \\
\hline Trickle Algorithm & Default \\
\hline Nodes rank & MRHOF + ETX Metrics \\
\hline DIO_Interval Min & $12 \mathrm{~ms}$ \\
\hline DIO_interval doublings & $8 \mathrm{~ms}$ \\
\hline
\end{tabular}

TABLE III. THE CONFIGURATION OF THE 6LBR PARAMETER

\begin{tabular}{|l|l|}
\hline Parameter & Setting \\
\hline Mode Selection & MODE=ROUTER \\
\hline $\begin{array}{l}\text { TAP mode - bridged or } \\
\text { tunneled to the Ethernet } \\
\text { network }\end{array}$ & RAW_ETH=0 \\
\hline Bridge Configuration & $\begin{array}{l}\text { BRIDGE=1 } \\
\text { CREATE_BRIDGE=1 } \\
\text { DEV_ETH=eth0 } \\
\text { DEV_BRIDGE=br0 } \\
\text { DEV_TAP=tap0 }\end{array}$ \\
\hline SLIP Radio configuration & $\begin{array}{l}\text { DEV_RADIO=/dev/ttyACM0 } \\
\text { BAUDRATE=115200 }\end{array}$ \\
\hline Configuration Files & $\begin{array}{l}\text { NVM=conf-templates/test.dat } \\
\text { CONFIG=/etc/6lbr/nvm.conf }\end{array}$ \\
\hline 6LBR installation paths & BIN_6LBR=\$LIB_6LBR/bin \\
\hline COOJA support & SOCK_RADIO=localhost \\
\hline
\end{tabular}

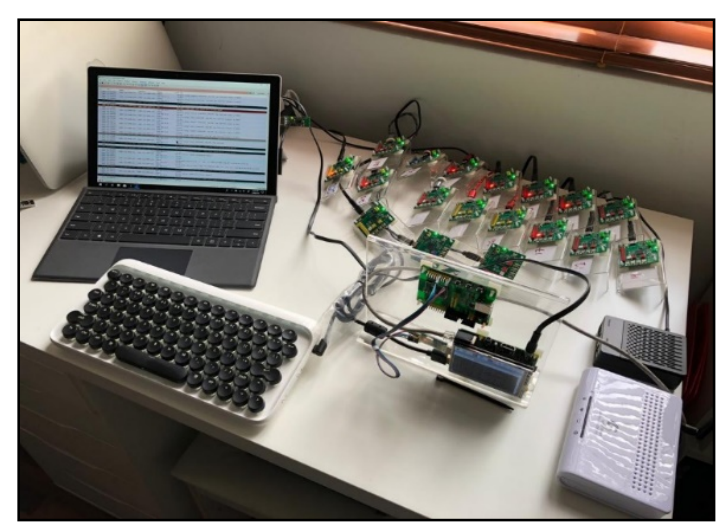

Fig. 8. Development of 6LoWPAN Testbed using CC2538 Mote.

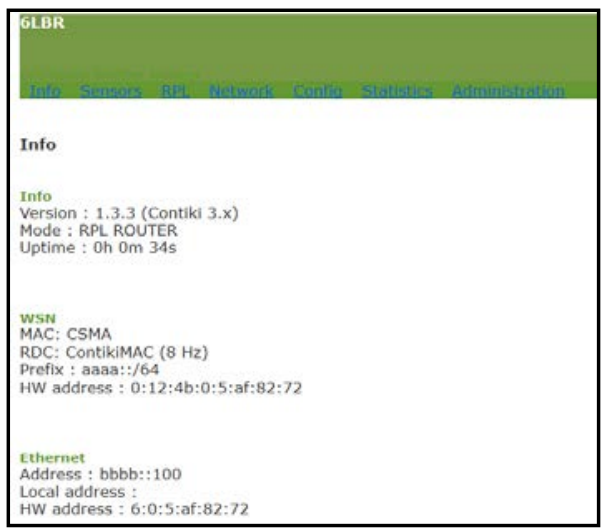

Fig. 9. Interface of 6LBR in Web Server. 


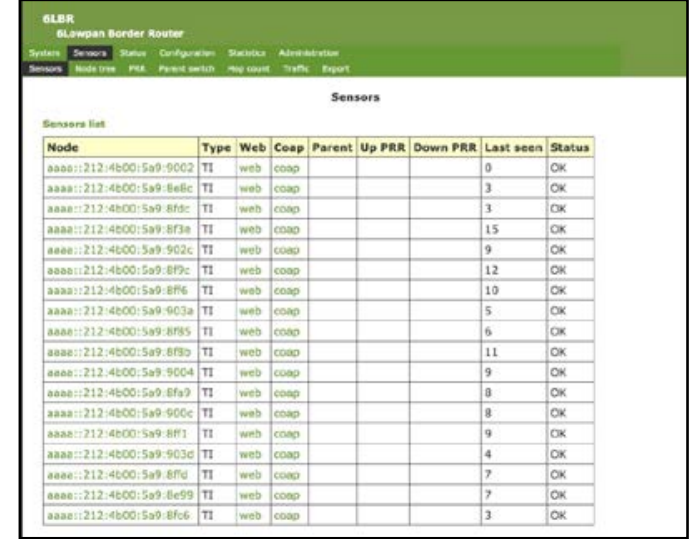

Fig. 10. Interface of Sensor Node in Web Server.

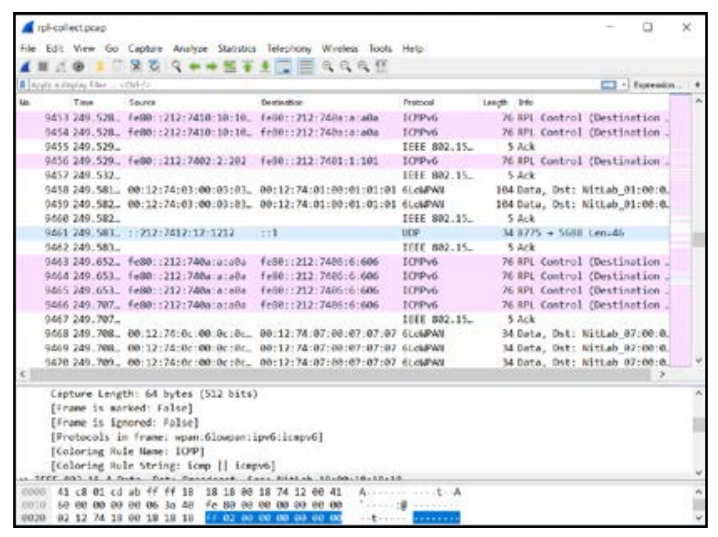

Fig. 11. Wireshark Capture Interface.

TABLE IV. ENERgY MODEL OF CC2538

\begin{tabular}{|l|l|}
\hline State & Current \\
\hline Active-Mode Listen (RX) & $20 \mathrm{~mA}$ \\
\hline Active-Mode Transmit TX at $0 \mathrm{dBm}$ & $24 \mathrm{~mA}$ \\
\hline
\end{tabular}

1) Performance Metric

2) Control Traffic Overhead (CTO): The total number of DIS, DIO, DAO message transmitted by nodes for the formation of DADOG in the network. CTO is calculated using the following equation (1) [25]-[27]:

CTO $=\sum_{k=1}^{n} D I S(k)+\sum_{k}^{n} D I O(k)+\sum_{k=1}^{n} D A O(k)$

3) Network Latency: Network Latency is defined as the difference of the taken time between the sending and the receiving of a packet via the DADOG path [25], [28]. The total of latency as mentioned in equation (2).

Total Latency $=\sum_{k=1}^{n}($ Received Time $(k)-$ Sent Time $(k)$

4) Convergence time (CT): CT is the total time needed for all the reachable nodes to join the DAG [29], [30] including the number of times to perform a global repair of the network after a link failure. In nutshell, CT is the process of building the DODAG from the first DIO messages sent until the last
DIO joined the DAG. CT calculated from the DIO message Equation (3) presents the formula to calculate the CT.

Convergence Time $=$ Last DIO joined DAGFirst DIO Send

\section{RESUlT AND DisCUSSION}

\section{A. Control Traffic Overhead}

Fig. 12 to 15 shows the comparison of the control message packets (DIS, DIO, DAO, CTO) between HRPL and RPL for each node after 30 minutes of the experiment time being. In these scenarios, HRPL sends $41.6 \%$ DIS, $36.2 \%$ DIO, and $40.8 \%$ DAO lesser than RPL. The result shows that HRPL significantly decreased $38.7 \%$ CTO packets on average compared to RPL as presented in Fig. 16. In fact, our observation shows, RPL generates too many control messages to build a DODAG route, which is used to propagate the routing tables between nodes. We also monitor that the DIO message packet was dominant the rest (DIS and DAO) of the RPL/HRPL control message packet in updating and construct the destination route. By enhancing the T-Cut delay (HRPL), a new DIO message will only operate based on $\mathrm{H}$ field conditions as long as the T-Cut delay is not over. On the other hand, this condition has reduced the retransmission of the DIO message. Indeed, our design methods present a better CTO by choosing the route path with the lowest cost and maximum PDR. Overall results conclude that the DIO, DIS, DAO, and CTO messages have been significantly reduced with the use of HRPL.

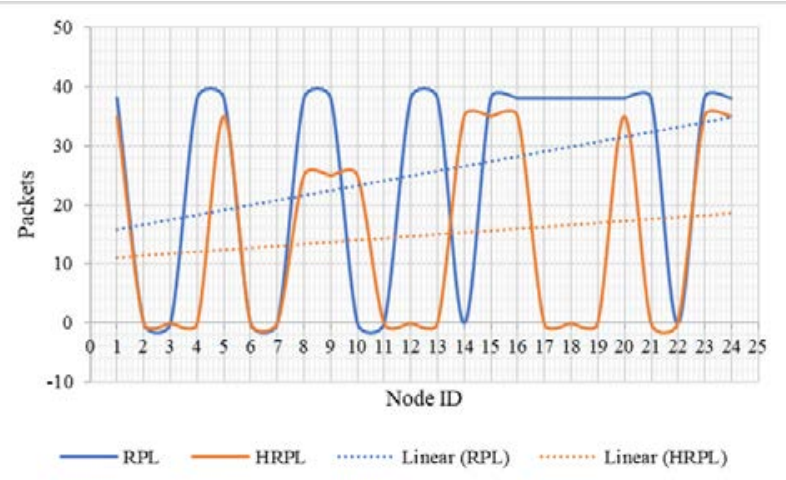

Fig. 12. DIS Messages for Each Node.

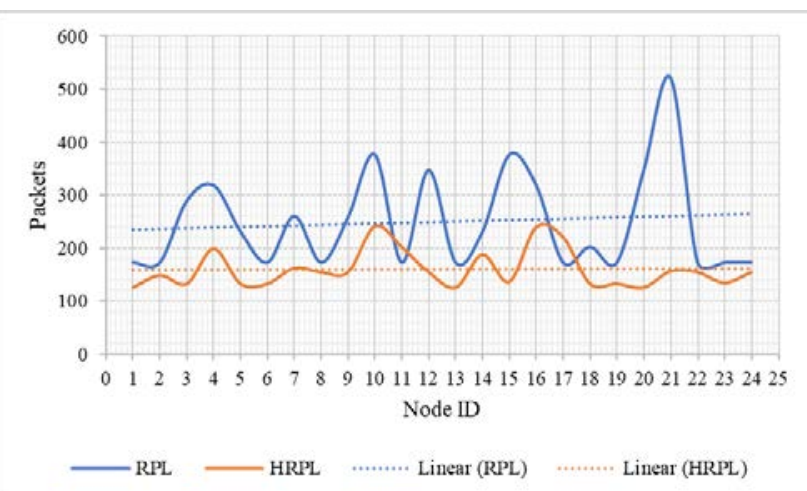

Fig. 13. DIO Messages for Each Node. 


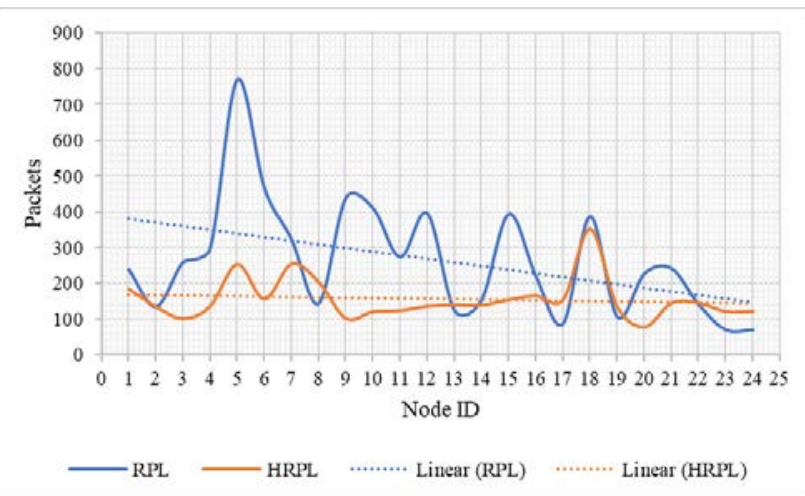

Fig. 14. DAO Messages for Each Node.

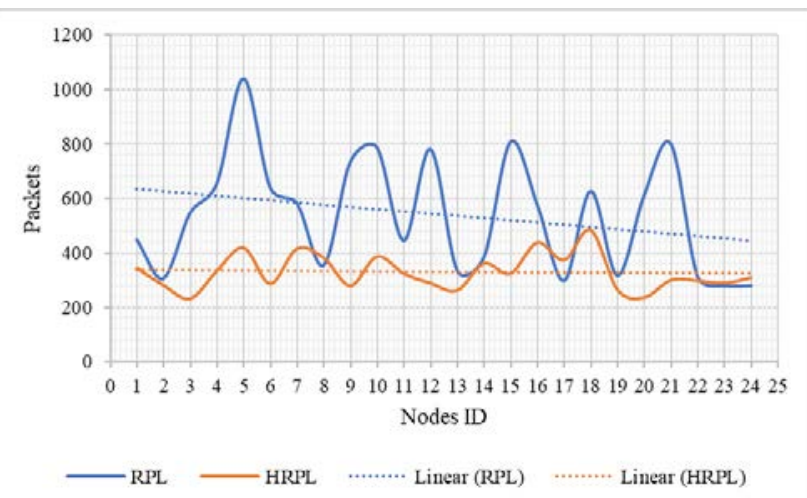

Fig. 15. CTO for Each Node.

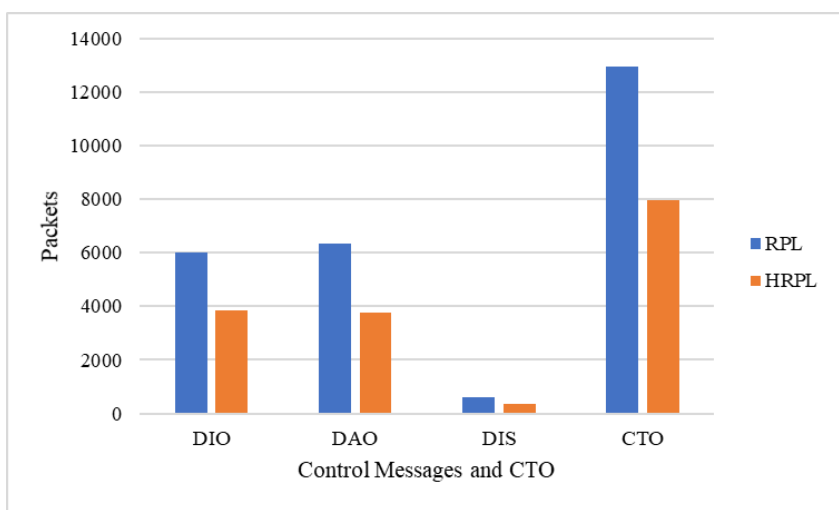

Fig. 16. Total of Control Messages and CTO.

\section{B. Network Latency}

Fig. 17 provides a comparison of the network latency between HRPL and RPL for each node. The test result shows that HRPL has lower latency (26\% lower) compared to RPL. The feature of the RPL radio duty cycle is the sender nodes send the DIO Message repeatedly until it gets the DIO Acknowledgment (DIO-ACK). The receiver nodes wake up to listen on packet transmission from the neighbor and still in radio on until transmission completes. By using the cut-off delay (HRPL), the delay for waiting the receiver wakes up has been minimized. This situation could reduce network latency.
It happens because the node with less packet still in radio on until the time of cut of delay expires. Due to this matter, the extra timing for the receiver wakes up on competing for the packet transmission is reduced. Whereas in RPL the less nodes go to radio off and it takes extra timing for switching back to radio on. These results are consistent with those of [11] who found that the radio duty cycle has an impact on the latency. These differences can be explained in part by the latencies for each node as presented in Fig. 18 (RPL) and Fig. 19 (HRPL). The total of RPL latency is $0.165 \mathrm{~s}$ and HRPL is $0.121 \mathrm{~s}$. We noticed that the network latency of HRPL is significantly reduced compared to RPL.

\section{Convergence Time}

As shown in Fig. 20, HRPL has better convergence time compared to RPL. HRPL shows $37.6 \%$ faster convergence time in this experiment. Due to this matter, the $\mathrm{H}$ condition in HRPL response was reducing the objective function (MRHOF-ETX) to take less time in selecting the best parent to join the DAG. At the same time, our approach successfully dropped the control message send across the network during the process of DODAG construction. In comparison with RPL, objective function took a longer time for RPL to build the DODAG, which may be caused by the complex process of objective function calculating the number of packet retransmission over the link.

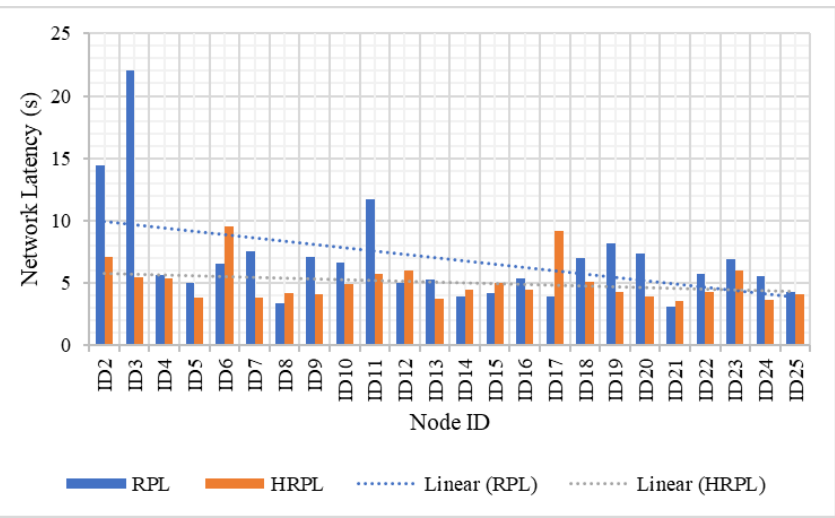

Fig. 17. Comparison of Latency between RPL and HRPL.

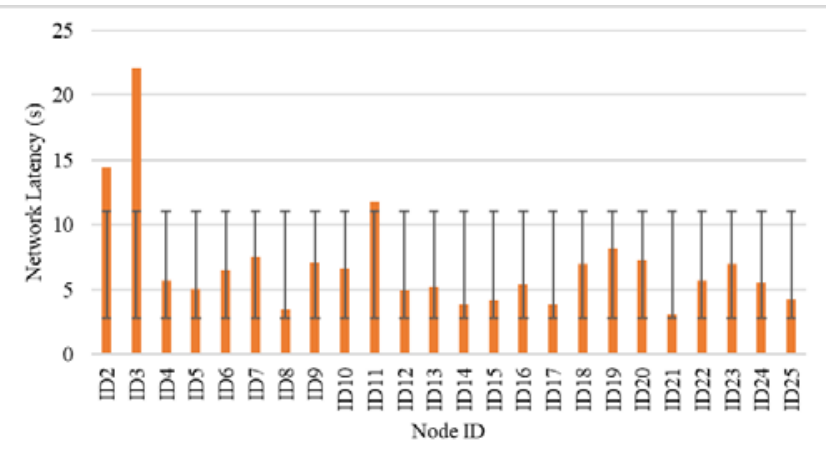

Fig. 18. The Total of RPL Latencies for Each Node. 


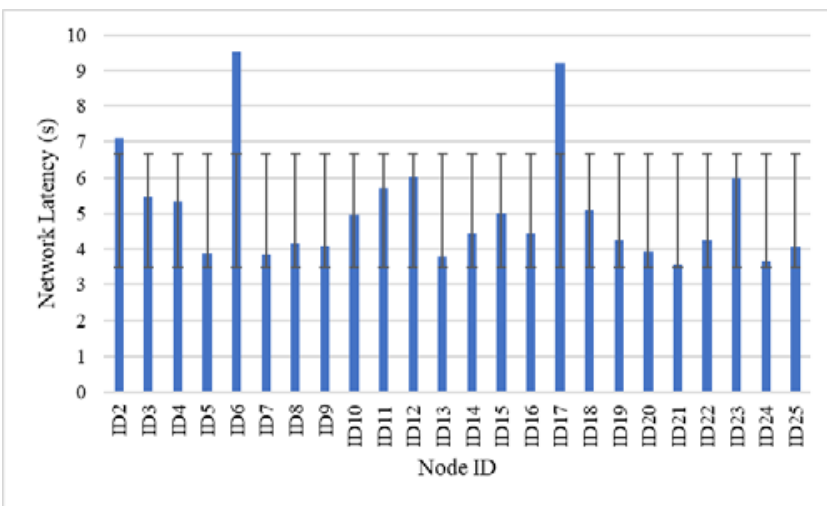

Fig. 19. The Total of HRPL Latencies for Each Node.

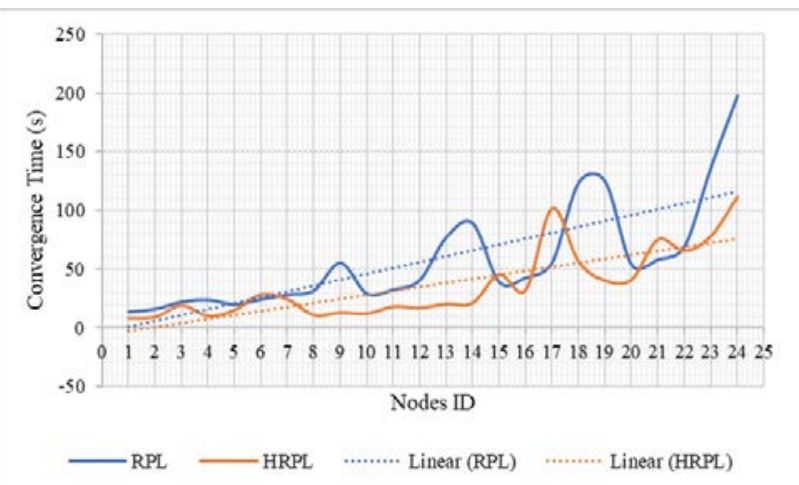

Fig. 20. RPL and HRPL Convergence Time for Each Node.

\section{CONCLUSIONS}

In this paper, a testbed has been developed to support physical experimentation which addresses the performance of our HRPL protocol in the real 6LoWPAN scenario. The result shows HRPL performs much better than RPL in all performance metrics that measured (CTO, latency, and convergence time). In conclusion, the testbed developed favorable result in implementing HRPL in a real scenario. However, still many issues could be researched especially the way to reduce the latency. It ended up wasting energy, as the receiver periodically needs to wake up to listen on packet transmissions from its neighbours. This phenomenon could be explained by the fact that in the UDP message transmission via IEEE 802.15.4 frame data, a packet is sent repeatedly until it gets an acknowledgment from the receiver. Further research should be done to investigate the HRPL in large-scale network in multiple topologies, and for a different number of nodes.

\section{ACKNOWLEDGMENT}

This work is conducted under the Fundamental Research Grant Scheme (FRGS) with reference number (FRGS/2018/FTMK-CACT/F00392) by the Ministry of Higher Education Malaysia and Universiti Teknikal Malaysia Melaka.

\section{REFERENCES}

[1] S. Ahmad and J. Zhang, "Network-State-Aware Quality of Service Provisioning for the Internet of Things,” Int. J. Adv. Comput. Sci. Appl., vol. 7, no. 6, pp. 369-376, 2016.

[2] S. El Bouanani, O. Achbarou, M. A. Kiram, and A. Outchakoucht, "Towards understanding internet of things security and its empirical vulnerabilities: A survey,” Int. J. Adv. Comput. Sci. Appl., vol. 10, no. 10, pp. 337-345, 2019.

[3] B. H. Malik et al., "Investigating technologies in decision based internet of things, internet of everythings and cloud computing for smart city," Int. J. Adv. Comput. Sci. Appl., vol. 10, no. 1, pp. 580-587, 2019.

[4] M. Gurunathan and M. A. Mahmoud, "A review and development methodology of a lightweight security model for IoT-based smart devices,” Int. J. Adv. Comput. Sci. Appl., vol. 11, no. 2, pp. 125-134, 2020.

[5] Y. Mahmood, N. Kama, A. Azmi, and S. Ya’acob, “An IoT based home automation integrated approach: Impact on society in sustainable development perspective,” Int. J. Adv. Comput. Sci. Appl., vol. 11, no. 1, pp. 240-250, 2020.

[6] R. F. Al-Mutawa and F. A. Eassa, "A smart home system based on internet of things,” Int. J. Adv. Comput. Sci. Appl., vol. 11, no. 2, pp. 260-267, 2020.

[7] Z. Shelby and C. Bormann, 6LoWPAN: The Wireless Embedded Internet. 2009.

[8] G. Keng Ee, C. Kyun Ng, N. Kamariah Noordin, and B. Mohd Ali, "A Review of 6LoWPAN Routing Protocols," Proc. Asia-Pacific Adv. Netw., vol. 30, pp. 71-81, 2010.

[9] J. J. P. C. Rodrigues and P. A. C. S. Neves, "A survey on IP-based wireless sensor network solutions,” Int. J. Commun. Syst., vol. 23, no. 5, pp. 633-652, 2010.

[10] R. Garg and S. Sharma, "A study on Need of Adaptation Layer in 6LoWPAN Protocol Stack,” I.J. Wirel. Microw. Technol., vol. 3, pp. 49-57, 2017.

[11] T. Zhang and X. Li, "Evaluating and analyzing the performance of RPL in contiki," in MSCC '14: Proceedings of the first international workshop on Mobile sensing, computing and communication, 2014, no. August 2014, pp. 19-24.

[12] H. Xie, G. Zhang, D. Su, P. Wang, and F. Zeng, "Performance evaluation of RPL routing protocol in Glowpan," in Proceedings of the IEEE International Conference on Software Engineering and Service Sciences, ICSESS, 2014.

[13] L. M. L. Oliveira, A. F. De Sousa, and J. J. P. C. Rodrigues, "Routing and mobility approaches in IPv6 over LoWPAN mesh networks," Int. J. Commun. Syst., vol. 24, no. 11, pp. 1445-1466, 2011.

[14] K. Naito, "A Survey on the Internet-of-Things : Standards, Challenges and Future Prospects,” J. Inf. Process. Vol.25, vol. 25, pp. 23-31, 2017.

[15] A. Saaidah, O. Almomani, L. Al-Qaisi, and M. K. Madi, "An efficient design of RPL objective function for routing in internet of things using fuzzy logic,” Int. J. Adv. Comput. Sci. Appl., vol. 10, no. 8, pp. 184190, 2019.

[16] J. Kantert, C. Ringwald, G. Von Zengen, S. Tomforde, L. Wolf, and C. Muller-Schloer, "Enhancing RPL for robust and efficient routing in challenging environments," Proc. - 2015 IEEE 9th Int. Conf. SelfAdaptive Self-Organizing Syst. Work. SASOW 2015, pp. 7-12, 2015.

[17] K. Iwanicki, "RNFD: Routing-Layer Detection of DODAG (Root) Node Failures in Low-Power Wireless Networks,” 2016 15th ACM/IEEE Int. Conf. Inf. Process. Sens. Networks, IPSN 2016 - Proc., 2016.

[18] N. H. Mohd Yusoff, N. A. Zakaria, and N. Harum, "Problem analysis of RPL overhead in 6LOWPAN using 5W1H model," Int. J. Innov. Technol. Explor. Eng., vol. 8, no. 12, pp. 5300-5305, 2019.

[19] N. H. Mohd Yusoff, N. A. Zakaria, A. Sikora, and J. S. Sebastian, "6LoWPAN Protocol in Fixed Environment: A Performance Assessment Analysis," Proc. 2019 10th IEEE Int. Conf. Intell. Data Acquis. Adv. Comput. Syst. Technol. Appl. IDAACS 2019, vol. 2, pp. 1142-1147, 2019.

[20] B. S. Hassen, S. A. S. Lafta, H. M. Noman, and A. H. Ali, "Analyzing the performances of WSNs routing protocols in grid- based clustering," Int. J. Adv. Sci. Eng. Inf. Technol., vol. 9, no. 4, pp. 1211-1216, 2019.

[21] P. Levis, T. H. Clausen, J. W. Hui, O. Gnawali, and J. Ko, "The Trickle Algorithm,” IETF RFC6206, 2011.

[22] N. Pradeska, Widyawan, W. Najib, and S. S. Kusumawardani, "Performance analysis of objective function MRHOF and OF0 in routing protocol RPL IPV6 over low power wireless personal area networks (6LoWPAN),” Proc. 2016 8th Int. Conf. Inf. Technol. Electr. 
Eng. Empower. Technol. Better Futur. ICITEE 2016, no. 7863270, p. 7863270, 2017.

[23] O. Gnawali and P. Levis, "The Minimum Rank with Hysteresis Objective Function,” IETF RFC6719.

[24] M. Banh, N. Nguyen, K. H. Phung, L. Nguyen, N. H. Thanh, and K. Steenhaut, "Energy balancing RPL-based routing for Internet of Things," 2016 IEEE 6th Int. Conf. Commun. Electron. IEEE ICCE 2016, pp. 125-130, 2016.

[25] H. Lamaazi and N. Benamar, "OF-EC: A novel energy consumption aware objective function for RPL based on fuzzy logic.," J. Netw. Comput. Appl., vol. 117, pp. 42-58, 2018.

[26] E. Ancillotti, R. Bruno, and M. Conti, "Reliable data delivery with the IETF routing protocol for low-power and lossy networks," IEEE Trans. Ind. Informatics, vol. 10, no. 3, pp. 1864-1877, 2014.
[27] B. Ghaleb, A. Al-dubai, E. Ekonomou, B. Paechter, and M. Qasem, "Trickle-Plus : Elastic Trickle Algorithm for Low- Power Networks and Internet of Things," in IEEE Wireless Communications and Networking Conference Workshops (WCNCW), 2016, pp. 103-108.

[28] J. Guo, P. Orlik, K. Parsons, K. Ishibashi, and D. Takita, "Resource aware routing protocol in heterogeneous wireless machine-to-machine networks,” 2015 IEEE Glob. Commun. Conf. GLOBECOM 2015, 2015.

[29] S. Biju and N. M. Shekokar, "Optimize the energy efficiency of RPL based 6LOWPAN by FL clustering,” 2017 Int. Conf. Big Data, IoT Data Sci. BID 2017, vol. 2018-Janua, pp. 159-166, 2018.

[30] C.-A. La, M. Heusse, and A. Duda, "Link reversal and reactive routing in Low Power and Lossy Networks,” IEEE Int. Symp. Pers. Indoor Mob. Radio Commun. PIMRC, pp. 3386-3390, 2013. 